\title{
The Education of the Greek Minority in Albania during Communism
}

\author{
Dr. Alketa Marku \\ Department of Administrative and Political Sciences, Faculty of Social Science \\ Albanian University-UFO, Tirana, Albania \\ E mail: a.marku@albanianuniversity.edu.al
}

\section{Doi:10.5901/jesr.2013.v3n7p232}

\begin{abstract}
During World War II and on, the greek-albanian relations were very tense, but as far as it concerns the situation of the greek minority in Albania the situation was quite different. This paper presents very shortly the situation faced by the Greek minority and the steps taken from the communist regime against the education of the greek minority in Albania, and it can be said that right after the war, Hoxha's government showed a special care and great attention to the education of the minority.
\end{abstract}

Keywords: greek minority, the education, communism, Albania

\section{Introduzione}

Nel secondo dopoguerra, si manifestò una tendenza contraria alla realizzazione di ulteriori forme di tutela internazionale delle minoranze, determinata dai risultati negativi tra le due guerre ${ }^{1} \mathrm{e}$ dall' influenza della dottrina americana del meltingpot. In base a questa, i diversi gruppi etnici devono rinunciare alle proprie particolarità, formando un'unica nuova nazionalità.

Questa impostazione condusse ad affermare che una volta assicurato il rispetto dei diritti individuali, non ci sarebbe bisogno di affermare i diritti delle minoranze 2

Con la stabilizzazione della situazione politica internazionale e l'evoluzione del pensiero politico si diffuse anche l'indirizzo pluralistico in base al quale la coesistenza tra gruppi diversi all'interno di uno Stato è possibile e il loro sviluppo si dovrà essere tollerato, e per di più favorito. Questo ha favorito un rinnovato impegno a favore della tutela delle minoranze non solo sul piano internazionale ma anche all'interno degli Stati.

\section{L'Albania del Dopo-Guerra}

Nel 1945 l'Albania diventa Repubblica Popolare, proclamata da Enver Hoxha, che impose pian piano dure riforme. Dall'inizio del suo regime vengono confiscate tutte le proprietà degli stranieri in Albania, vengono statalizzate tutte le istituzioni industriali e commerciali, viene imposto il monopolio sia per il commercio interno che estero. II governo proclama la riforma agraria, statalizzando tutte le foreste, confiscando tutte le proprietà. La costituzione approvata nel 1946 garantisce la libertà di religione, ma già dall'inizio i comunisti dimostrano la loro intenzione che in un futuro non molto lontano avrebbero impedito ogni tipo di organizzazione religiosa. Le tre principali religioni: musulmana, ortodossa e cattolica divengono oggetto di molte discriminazioni da parte del governo, che usa la violenza ed il terrore per sottometterle. Le proprietà appartenenti alle comunità religiose vengono confiscate. Agli inizi degli anni ' 50 molti tra gli importanti capi religiosi vengono fucilati, imprigionati o esiliati. La situazione si aggrava negli anni '50-' 60 per arrivare al 1967, quando con una delibera del governo vengono chiuse tutte le chiese e le moschee, e la libertà di religione è soppressa. Questa elite è nuova e senza esperienze; con l'aggravarsi del conflitto tra occidente ed oriente, il regime di Hoxha lega col blocco sovietico distanziandosi dall'occidente e soprattutto dai paesi vicini come Grecia e Italia (Biberaj, 2000).

\footnotetext{
${ }^{1}$ Secondo diversi autori il sistema di tutela di minoranze etniche, creatosi dopo la prima guerra e patrocinato dalla Lega delle Nazioni si rivelò poco efficace.

2 Basandosi alla dichiarazione della signora Eleanor Roosvelt, allora presidente della Commissione dell'Onu per i diritti dell'uomo.
} 


\section{La Situazione della Minoranza Greca durante il Periodo Comunista}

Già dal secondo conflitto mondiale e nel periodo a seguire, le relazioni greco-albanesi diventano critiche. La tensione era iniziata già dall'occupazione italiana del 1939, ed aveva come scopo la riattivazione e la rinascita della politica dell' annessione dei territori dell'Albania del Sud, una politica uguale a quella seguita prima e durante il primo conflitto mondiale dal governo greco. A questa tensione si aggiunge, dopo il 1946, un nuovo elemento, che riguardava alla "guerra civile in Grecia" ed il sostegno del governo comunista (albanese) alle guerriglie greche. Con la fine della "guerra civile" in Grecia nell' estate del 1949 la tensione tra i due paesi è meno forte, ma i rapporti sono ancora troppo freddi. Durante gli anni a seguire la politica del governo albanese tenta ad un riavvicinamento ma senza successo, e solo nel 1971 si stabiliscono i rapporti diplomatici (Meta, 2004). Cosi negli anni a seguire, specialmente verso gli anni ' 80 molti sono gli accordi bilaterali: nel 1984 vengono stipulati cinque accordi che comprendevano; gli scambi culturali, le comunicazioni, le poste, la cooperazione scientifico-tecnologica ed il trasporto. A gennaio del 1985 viene aperto il punto doganale di Kakavija, il che dava possibilità di collegamento alla minoranza greca con la Grecia.

\section{L'istruzione}

In Albania, già dal 1945, per quanto riguarda l'istruzione, viene inserito nella Costituzione il diritto dei cittadini di entrambi i sessi ad un'educazione gratuita. Diverse riforme vengono poi elaborate nel corso degli anni. La riforma del 1946 anno in cui l'Albania viene proclamata repubblica popolare (poi, nel 1976, "repubblica popolare socialista") - istituisce un sistema scolastico unico e obbligatorio. Le scuole vengono separate dalle chiese e viene decretato, per le minoranze nazionali greca e macedone, il diritto di ricevere l'istruzione nella propria lingua.

Gia dall'inizio il regime aveva dimostrato una grande attenzione sull'istruzione in lingua greca. Cosi nell'anno accademico 1949/50 nelle regioni di Argirocastro e Saranda, dove era situata la minoranza greca, esistevano 9 scuole uniche (I-VIII) per la minoranza greca con 618 alluni e 77 scuole elementari (I-IV) con 4159 alluni, quando la popolazione della minoranza greca, in base al censimento della popolazione albanese del 1945, ammontava a 24.191 persone, 11.881 nella regione di Argirocastro e 12.310 nella regione di Saranda 3 .

La riforma del 1963 rafforza la dimensione politecnica dell'educazione, mentre quella del 1969 ne consolida il rapporto con il mondo del lavoro 4 .

Nel 1969, Enver Hoxha contesta in modo categorico, davanti alla Byrò Politica del PPSH (Partito del Lavoro Albanese) la proposta sulla chiusura del giornale della minoranza greca "Laiko Vima", ritenendola una decisione sbagliata e di carattere sciovinista. In questo discorso Hoxha raccomanda e chiede insistentemente la conservazione, l'appoggio ed il rafforzamento delle scuole elementari greche della minoranza, lo sviluppo della cultura greca e la conservazione della compatezza nazionale della minoranza - accentuando che nelle scuole della minoranza greca "si deve continuare ad insegnare con serietà e rigorosità la lingua greca"(Meta, 2004).

L'opinione degli studiosi greci era che il governo albanese aveva cercato di creare una identità diversa e nuova per la minoranza greca, criticando soprattutto il contenuto dei testi scolastici. E come afferma Evangelos Kofos (esperto greco dei Balcani), nei testi scolastici degli anni '50 esisteva materiale di carattere patriotico greco, il che influiva sullo sviluppo delle relazioni culturali e sentimentali tra la Grecia e la comunità di etnia greca in Albania. Kofos è critico, invece, verso i testi degli anni 1970-72, ritenendo che avevano perso questi valori (Kofos, 1961). Ma bisogna dire che tali misure furono prese per tutti i cittadini ( soprattutto dopo la chiusura dei luoghi di culto e l'abbolizione di tutti i nomi sacri ecc.) e non avevano nessun carattere o scopo specifico contro la minoranza greca. Kofos distingue un terzo periodo, quello degli anni 1973-75; in questo periodo nei testi veniva notato un modo diverso nel trattare le relazioni greco-albanesi (inizio dei rapporti diplomatici dopo 1971) riguardo a quello precedente. Oltre a quello sopra menzionato, sulla situazione della minoranza greca e della chiesa ortodossa durante questo periodo non esistono fonti o statistiche precise; con la caduta del comunismo, negli anni ' 90 , l'Albania diventa preda di un caos totale che porta alla distruzione di quasi qualsiasi oggetto che apparteneva al regime (la maggior parte dagli stessi ex comunisti per cancellare ogni prova), perciò in Albania è molto difficile indagare 0 avere delle statistiche di quegli anni.

\footnotetext{
${ }^{3}$ AMPJ, viti 1950, D.B./VII-3. Statistike mbi minoritetin grek ne Shqiperi dhe mbi çamerine. (Archivio MAE - Statistiche sulla minoranza greca in Albania e sulla regione di çameria).

4 La scuola in Albania : Unesco, rapport mondial sur l'èducation, 1995
} 


\section{Conclusioni}

Durante il periodo del comunismo, la situazione della minoranza greca, specialmente per quanto riguarda l'istruzione, fu in grandi linee uguale a quella della popolazione albanese, non avendo subito alcun tipo di discriminazione specifica come minoranza. Anche l'esperto greco dei Balcani, Kofos, in uno dei suoi articoli affermo' che: "I'Albania ha le problematiche minori con le minoranze in confronto a tutti gli altri paesi comunisti della penisola balcanica".

\section{References}

AMPJ- Archivio del Ministero degli Affari Esteri (Albania)

Biagini A., Storia dell'Albania, dalle origini ai nostri giorni, Bompiani, Milano, 2007.

Biberaj E., Shqiperia ne tranzicon ORA, Tirana 2000, pg, 45-46

Meta B., Shqiperia dhe Greqia, 1949-1990, Paqja e veshtire Tirane 2004, pg. 9-

La scuola in Albania : Unesco, rapport mondial sur l'èducation, 1995

Kofos,E., Balcan Minorities Under Communist Regimes, in Balcan Studies", vol.2, nr.1, Thesaloniki 1961, pg.42.

Smirnova N., Historia e Shqiperise pergjate shekullit XX IDEart, Tirana 2004.

R.M Della Rocca Shqiperia rrenjet e Krizes, Tirana 2000

S.Tchirkovitch, La regle de non-discrimination et la protection des minoritès, in Revue general de droit international, 1951, pg, 259.

G.Malinverni, II progetto di Convenzione per la protezione delle minoranze elaborato dalla Commissione europea per la democrazia attraverso il diritto, in Rivista internazionale dei diritti dell'uomo,Anno $V \mathrm{n}^{\circ}$ 1, gennaio- aprile 1992, pg, 120. 